\title{
The interplay of thermodynamics and kinetics in dictating organocatalytic reactivity and selectivity
}

DOI:

10.1351/pac-con-13-01-14

Link to publication record in Manchester Research Explorer

\section{Citation for published version (APA):}

Burés, J., Armstrong, A., \& Blackmond, D. G. (2013). The interplay of thermodynamics and kinetics in dictating organocatalytic reactivity and selectivity. Pure and Applied Chemistry, 85. https://doi.org/10.1351/pac-con-13-0114

\section{Published in:}

Pure and Applied Chemistry

\section{Citing this paper}

Please note that where the full-text provided on Manchester Research Explorer is the Author Accepted Manuscript or Proof version this may differ from the final Published version. If citing, it is advised that you check and use the publisher's definitive version.

\section{General rights}

Copyright and moral rights for the publications made accessible in the Research Explorer are retained by the authors and/or other copyright owners and it is a condition of accessing publications that users recognise and abide by the legal requirements associated with these rights.

\section{Takedown policy}

If you believe that this document breaches copyright please refer to the University of Manchester's Takedown Procedures [http://man.ac.uk/04Y6Bo] or contact uml.scholarlycommunications@manchester.ac.uk providing relevant details, so we can investigate your claim.

\section{OPEN ACCESS}


Pure Appl. Chem., Vol. 85, No. 10, pp. 1919-1934, 2013.

http://dx.doi.org/10.1351/PAC-CON-13-01-14

(C) 2013 IUPAC, Publication date (Web): 29 July 2013

\title{
The interplay of thermodynamics and kinetics in dictating organocatalytic reactivity and selectivity*
}

\author{
Jordi Burés ${ }^{1,2}$, Alan Armstrong ${ }^{2}$, and Donna G. Blackmond ${ }^{1,2, \ddagger}$ \\ ${ }^{1}$ Department of Chemistry, The Scripps Research Institute, La Jolla, CA 92037, \\ USA; ${ }^{2}$ Department of Chemistry, Imperial College London, London SW7 2AZ, UK
}

\begin{abstract}
Recent reports of the real-time identification of intermediates in organocatalytic reactions by NMR spectroscopy coupled with detailed kinetic studies highlight a potential role for stable intermediates reversibly formed downstream from what is generally considered to be the enantioselectivity-determining step. In this work, we employ kinetic modeling to explore these concepts further. We demonstrate that when an intermediate is common to multiple reaction pathways, the relative reactivity of these pathways dictates the ultimate outcome, regardless of the relative stability of other intermediates connected to these pathways. Kinetic modeling also illustrates important implications for enantioselectivity depending on whether such intermediates lie on or off the catalytic cycle.
\end{abstract}

Keywords: asymmetric catalysis; catalysis; kinetics; mechanisms; modeling; reaction thermodynamics.

\section{INTRODUCTION}

In situ monitoring of reactive species has contributed to recent advances in the mechanistic understanding of organocatalytic reactions [1-3]. Since Gschwind and co-workers first identified enamines formed between aldehydes and proline [1a], NMR spectroscopy has proven to be a tool vital to these investigations, which have been expanded to include other pyrrolidine-based catalysts. All of this recent work owes a large debt to early pioneering studies of the stoichiometric reactions of enamines by a number of groups, including Seebach and co-workers [4], spanning a half-century of research [5].

One of the most successful organocatalytic transformations reported to date has been the conjugate addition of aldehydes to nitro-olefins, in particular reactions catalyzed by systems lacking a Brønsted acid co-catalyst such as the diarylprolinol ethers introduced by Hayashi [6] and by Jorgensen [7] (Scheme 1). Indeed, this transformation has become a benchmark reaction for assessing the performance of new organocatalysts [8].

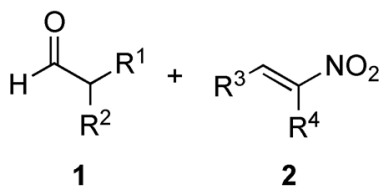

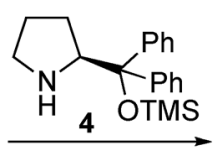

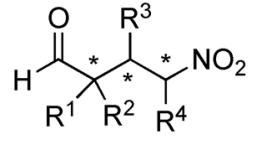

3

Scheme 1 Conjugate addition of aldehydes to nitro-olefins catalyzed by diarylprolinol ethers.

\footnotetext{
*Pure Appl. Chem. 85, 1919-2004 (2013). A collection of invited papers based on presentations at the $21^{\text {st }}$ International Conference on Physical Organic Chemistry (ICPOC-21), Durham, UK, 9-13 September 2012.

¥Corresponding author
} 
The accepted mechanistic paradigm in enamine catalysis for these systems relies on steric considerations as shown in Scheme 2. It is thought that bulky substituents on the pyrrolidine ring block one face of the enamine, making the approach of an electrophile more facile from the other face. In this steric model, the enantio-determining transition state derives from this interaction between the enamine and electrophile [9]. However, recent investigations have suggested that this model may be inadequate to account for a number of the experimental observations in the Michael addition to nitro-olefins as well as in a variety of other reactions effected by diarylprolinol ether catalysts. Most significantly, our group [2a] and that of Seebach with Hayashi [3a] separately found that the resting state [10] in the catalytic reaction of Scheme 1 is a cyclobutane species that occurs subsequent to the step shown in Scheme 2. The formation of this species, as well as the electrophile attack step shown in Scheme 2, were found to be rapidly reversible. This reversibility suggests that the stereochemical outcome may not in fact be determined by the transition-state model shown in Scheme 2, highlighting a potential role for "downstream species" in dictating the ultimate product selectivity. Further studies have expanded on these concepts $[2,3,11]$.

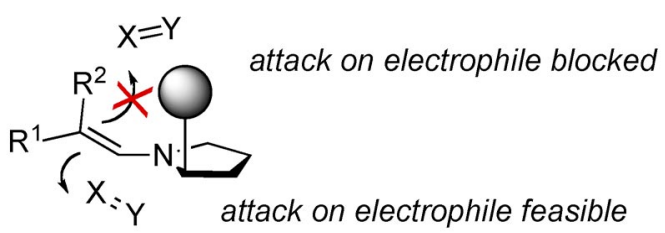

Scheme 2 Steric transition-state model for attack of a pyrrolidine-based enamine on an electrophile $\mathrm{X}=\mathrm{Y}$ for catalyst systems lacking a Brønsted acid co-catalyst.

The purpose of the present work is to employ kinetic modeling of selected reaction scenarios to help clarify the relative roles of kinetics and thermodynamics in cases where downstream species may be of consequence in the reaction mechanism. The main conclusions may be summarized as follows:

- When an intermediate species is common to several potential reaction pathways, the rate of each pathway depends equally on the concentration of the common intermediate, regardless of the relative stability of the products formed along each pathway. Thus, a low rate of product formation must be attributed to a low rate constant rather than to a fleeting concentration of the common intermediate.

- Highly stable intermediate species may be highly dynamic, with rapid formation and dissociation rates.

- When two intermediates are reversibly connected but react along parallel pathways to form two products, a low rate of product formation on one of the pathways compared to the other may be attributed to either (or both) a low rate constant and/or low intermediate concentration.

- The concentration of an intermediate species reversibly connected off-cycle to a steady-state catalytic network with two parallel pathways can have no influence on the relative selectivity of those pathways.

- The concentration of an intermediate species reversibly connected on-cycle to a steady-state catalytic network with two parallel pathways can have a profound influence on the selectivity of those pathways.

While some of these conclusions may not be intuitively obvious, they stem from basic principles describing the thermodynamics and kinetics of reaction networks. The reaction simulations leading to these conclusions are presented and discussed below. 


\section{BACKGROUND}

A recent study by Seebach and co-workers focused on the isolation and stoichiometric reaction of cyclic intermediates formed between enamines of aldehydes with diphenylprolinol trimethylsilyl (TMS) ether and nitrostyrenes [3b]. Stable cyclobutanes and 1,2- $N$-oxazines were observed by NMR spectroscopy, and in some cases crystal structures were determined. Although the zwitterion shown in Scheme 3 is not observed experimentally, this species is thought to play a central role, forming in the initial interaction between the enamine and the nitro-olefin and undergoing reactions in subsequent steps to give downstream species both on and off the catalytic cycle.

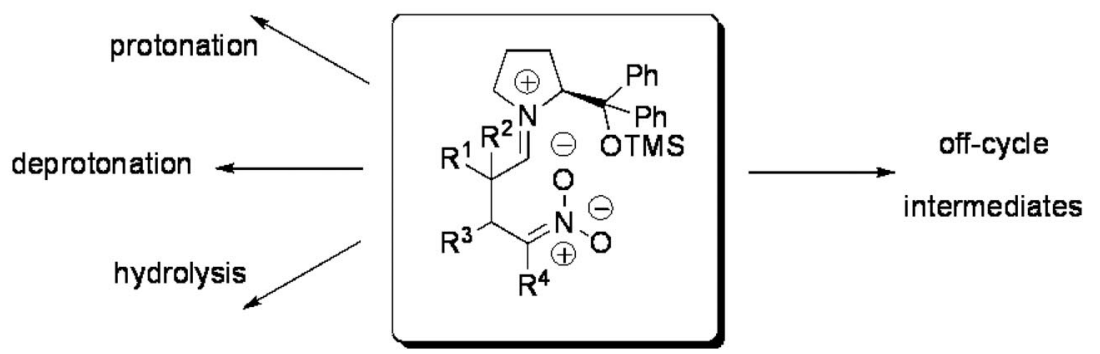

Scheme 3 Central role of zwitterionic species in proposed reactions on the catalytic cycle (left) and in formation of off-cycle species (right).

In the case of linear aldehydes $\left(\mathrm{R}^{2}=\mathrm{H}\right)$ and $\alpha$-substituted nitro-olefins $\left(\mathrm{R}^{4} \neq \mathrm{H}\right)$ in stoichiometric reactions with 4 , Seebach found that the six-membered ring intermediates were favored over the cyclobutane species for both propanal and for isovaleraldehyde (Scheme 4). Interestingly, addition of the nitro-olefin was found to be irreversible for the former aldehyde and reversible for the latter.

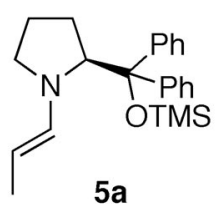

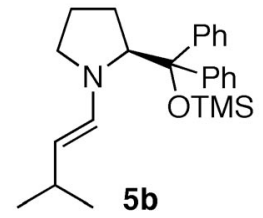

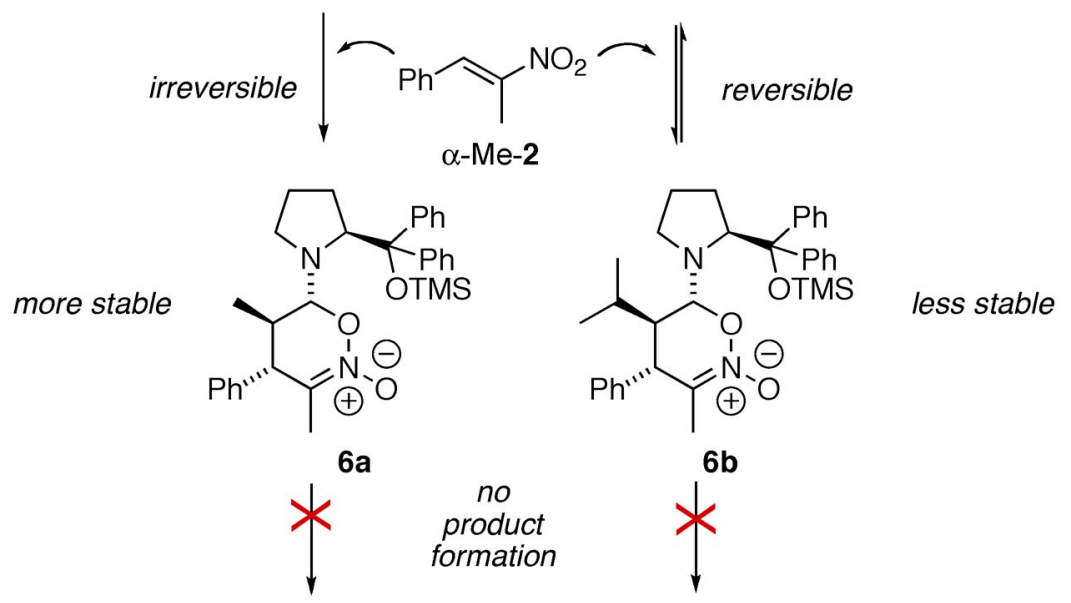

Scheme 4 Stable intermediates identified in stoichiometric studies by Seebach and co-workers. 
Turnover of the catalyst, with formation of the nitroaldehyde reaction product, was not observed in either case. The authors proposed that the failure of the catalytic reaction may be due not to a lack of reactivity of species, but instead may be attributed to the high stability of the catalyst resting states. Observation of a much higher stability for $\mathbf{6 a}$ compared to $\mathbf{6 b}$ was correlated with its irreversible formation, with the proposal that such species serve as an off-cycle trap in which the catalyst "rests forever" [3a].

Such correlations between stability and reactivity of intermediates have been shown to have implications beyond catalyst turnover, also influencing selectivity in competitive pathways. For example, the concept of high stability corresponding with low reactivity figured prominently in the mechanistic rationalization of asymmetric hydrogenation of enamides by chiral Rh complexes studied by Landis and Halpern [12]. Two diastereomeric intermediates were found to be present in a 10:1 ratio while enantioselectivities of $98 \%$ in excess of the product derived from the minor diastereomer were obtained. The term "major-minor" paradigm has been coined to describe this scenario. Another unusual selectivity paradigm termed "monopolizing kinetics" has been delineated in competitive reactions where a more stable but less reactive intermediate leads to the major product by effectively monopolizing all of the catalyst in the slower pathway [13]. Both of these scenarios are in contrast with the "lockand-key" mechanism conventionally associated with enzyme catalysis, where the most stable intermediate is also most active, leading to the dominant product. All of these cases may be rationalized by the Curtin-Hammett principle [14], which reveals that both the relative stabilities and relative reactivities of interconverting intermediates may contribute, in varying degrees in different reactions and under different circumstances, to the ultimate selectivity achieved in competitive reaction pathways.

Our own studies in the Michael addition of aldehydes to nitro-olefins using diphenylprolinol ether as catalyst revealed interesting features concerning the stability and reactivity of intermediates [2]. Both linear $\left(\mathrm{R}^{2}=\mathrm{H}\right)$ and $\alpha$-branched $\left(\mathrm{R}^{2} \neq \mathrm{H}\right)$ aldehydes were observed to form stable cyclobutane intermediates such as 7a and 7c (Scheme 5). In each case the cyclobutane was formed as a single diastereomer, in rapid, quantitative, and reversible fashion. Detailed kinetic studies showed that the entire cata-

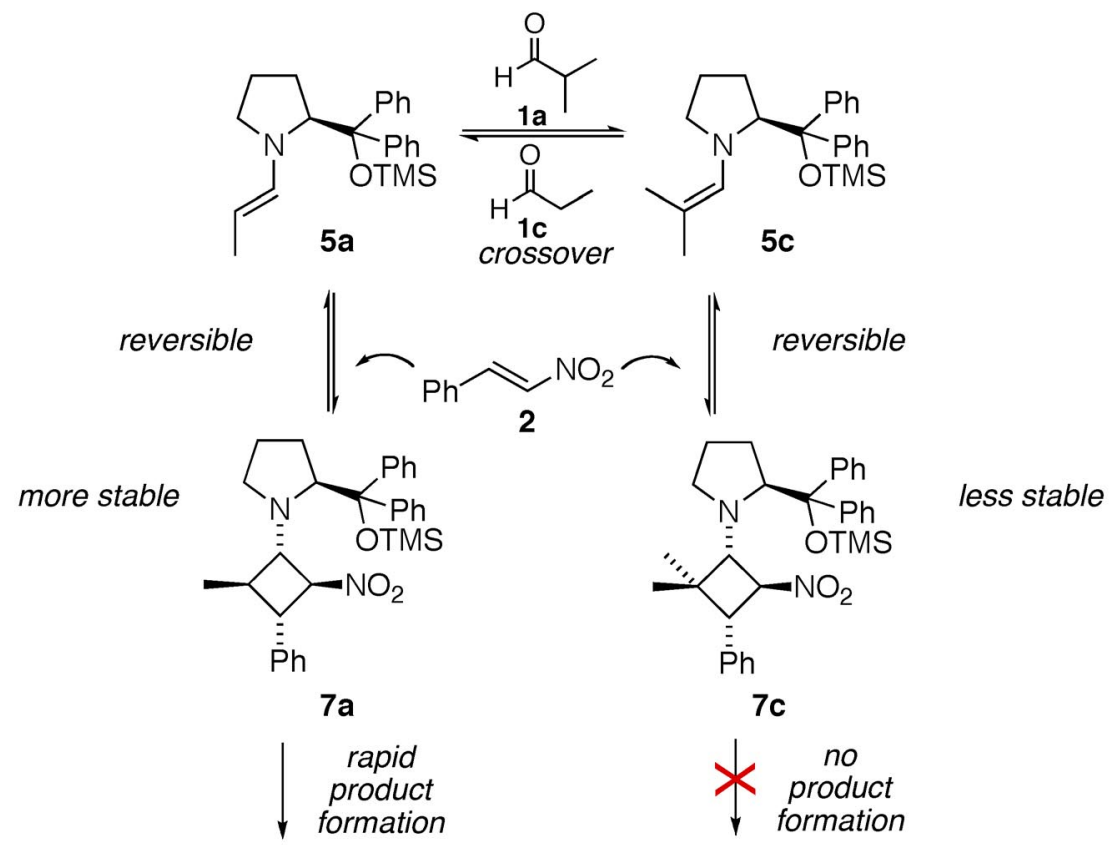

Scheme 5 Stable intermediates identified in catalysis and crossover studies by Burés et al. 
lyst concentration was effectively sequestered as the cyclobutane within minutes of introducing the reactants to the catalyst. If conversion to the product nitroaldehyde proceeds, it must therefore derive from this species, either by retrieving the off-cycle species back onto the cycle or by reacting directly from this species existing on the cycle itself. In fact, catalytic turnover was observed only for the reaction with linear aldehydes (Scheme 5), with cyclobutane 7c acting as an inert resting state $(<5 \%$ conversion in $24 \mathrm{~h}$ ) preventing turnover to the $\alpha$-branched nitroaldehyde product. However, further experiments revealed not only was the catalyst not "trapped forever" as 7c, but that this species undergoes facile interconversion with 7a in crossover experiments, with the unreactive species $\mathbf{7 c}$ found to be less stable than the catalytically active 7a (see Supporting Information for ref. [2a]). Thus, a possible reason for the low reactivity of $\alpha$-substituted aldehydes to form product nitroaldehydes cannot be attributed to species such $\mathbf{7} \mathbf{c}$ being too stable to ring-open, as Seebach suggested [3a], and as was reiterated recently by Moberg [8]. Thus, high stability in the formation of downstream intermediates is not necessarily correlated with low catalytic turnover.

These results suggest the need to develop a better understanding of relationships between thermodynamics (stability of intermediates) and kinetics (reactivity of intermediates) in systems featuring downstream intermediates. Of particular interest is the central role played by species such as the zwitterion of Scheme 3, which may serve as a junction connecting a number of possible reaction pathways. We probe these aspects further by turning to kinetic simulations [15] based on reaction scenarios reported experimentally such as those of Schemes 4 and 5. The results of these simulations and their mechanistic implications are discussed in the next section.

\section{RESULTS AND DISCUSSION}

\section{Reactivity of zwitterions}

\section{Stoichiometric reactions arising from zwitterions}

While Schemes 4 and 5 show experimentally observed downstream species directly formed from the reaction between enamine and nitro-olefin, implicit in these reactions is the intermediacy of unobserved species such as the zwitterion shown in Scheme 3. Such a species is likely to be formed initially from reaction between enamine and nitro-olefin, even if its low steady-state concentration precludes its experimental identification in most cases. Scheme 6 shows a simplified network of reaction steps explicitly centered around a zwitterion according to the stoichiometric transformations described by Seebach and co-workers [3b]. In the case of the isovaleraldehyde enamine $\mathbf{5 b}$ shown in Scheme 4, reaction with $\alpha$-methyl-substituted nitro-olefin $\alpha-\mathrm{Me}-2$ is suggested to lead to reversible formation of a zwitterion that reacts further, reversibly, to form heterocycle $\mathbf{6 b}$. The pathway to the nitroaldehyde prod-

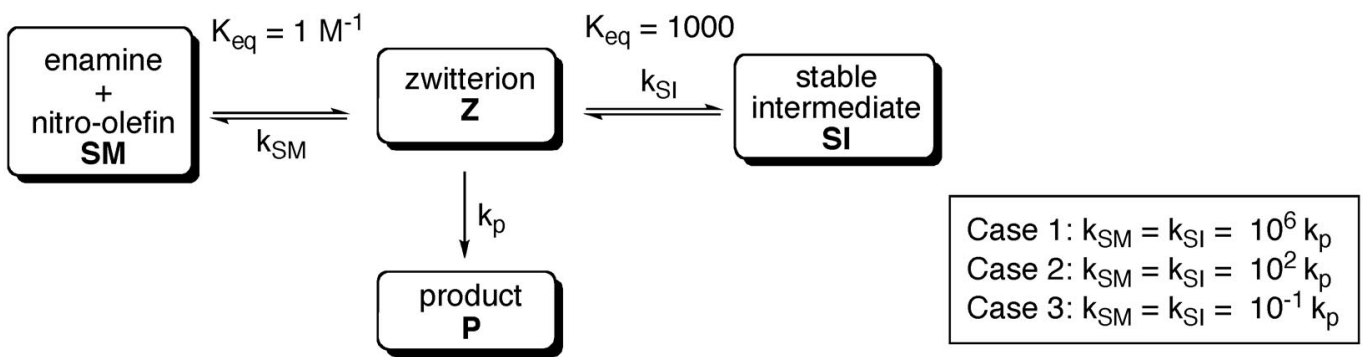

Scheme 6 Network detailing a central role for reactions from zwitterion Z: (i) back to starting materials $\mathbf{S M}$ via $k_{\mathbf{S M}}$; (ii) formation of stable cyclic intermediate SI via $k_{\mathbf{S I}}$; (iii) formation of catalytic reaction product $\mathbf{P}$ via $k_{\mathrm{p}}$. Simulations are carried out for three cases with $k_{\mathbf{S M}}=k_{\mathbf{S I}}$ set at different values relative to $k_{\mathrm{p}}$. Regeneration of the catalyst and the presence of other species potentially involved in the network, such as water or acid additives, are omitted for simplicity. 
uct presumably would also pass through this zwitterion, as in the general outline of Scheme 6. Lack of product formation under catalytic conditions was rationalized by these authors with the suggestion that the relative magnitude of the product-forming reaction rate (rate constant $k_{\mathrm{p}}$ in Scheme 6) is very low compared to those of the other two pathways emanating from the zwitterion, namely, its collapse to the heterocycle SI (rate constant $k_{\mathbf{S I}}$ in Scheme 6) and its dissociation back to starting materials SM, enamine and nitrostyrene (rate constant $k_{\mathbf{S M}}$ in Scheme 6). The failure of the reaction to form product $\mathbf{P}$ was proposed to be due not simply to lack of reactivity (low $k_{\mathrm{p}}$ ), but to the high stability (high $K_{\mathrm{eq}, \mathbf{S I}}$ ) of the off-cycle heterocycle SI. Thus, the rate of product formation is suggested to be influenced by the rates of other reactions with the zwitterion $\mathbf{Z}$ as a reactant.

Reaction simulations were carried out based on the three different scenarios of relative reactivity in the stoichiometric reaction network shown in Scheme 6. In each case, the equilibrium constant for formation of $\mathbf{Z}$ from enamine and nitro-olefin is set at $K=1 \mathrm{M}^{-1}$, in keeping with expectation of the low $\mathbf{Z}$ concentration compared to that of other species. In like manner, the equilibrium constant for formation of the stable off-cycle intermediate SI is set at $K_{\text {eq,SI }}=1000$ because of its experimentally observed high stability. With these parameters, the concentration of the zwitterion never exceeds $0.1 \%$ of the total catalyst concentration. For simulations of cases 1-3 in Scheme 6, the product rate constant $k_{\mathrm{p}}$ is fixed, while the other two rate constants for reactions emanating from the zwitterion are set equal $\left(k_{\mathbf{S I}}=k_{\mathbf{S M}}\right)$ and are given values relative to $k_{\mathrm{P}}$ spanning a range of seven orders of magnitude, from one million fold greater (case 1) to tenfold smaller (case 3). This range allows us to test Seebach's hypothesis that much faster rates back to $\mathbf{S M}$ or off-cycle to $\mathbf{S I}$ are responsible for preventing the formation of product $\mathbf{P}$. The results of these simulations are shown in Fig. 1.
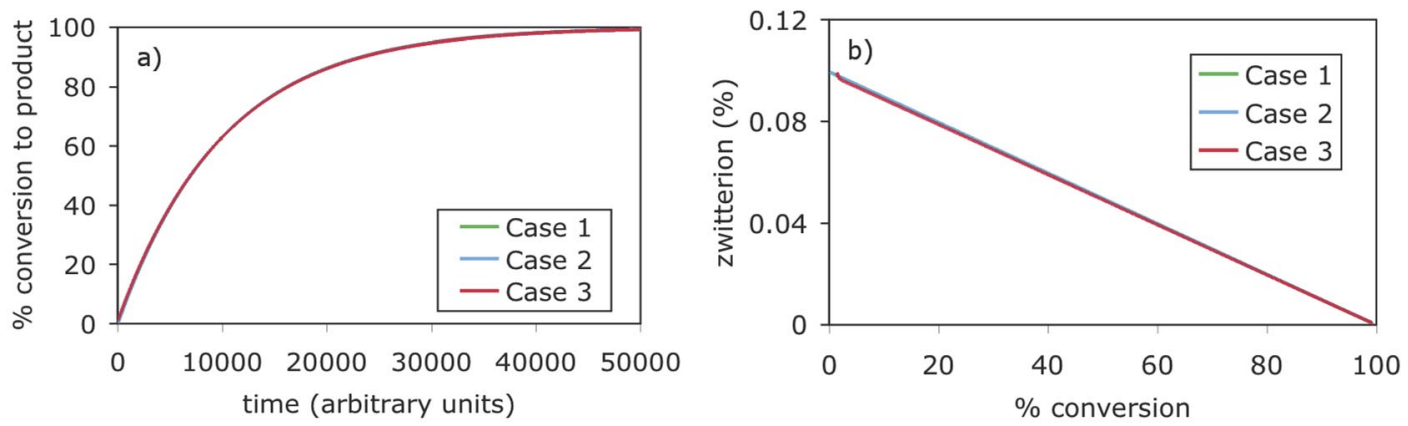

Fig. 1 Kinetic simulations of the reaction network shown in Scheme 6 for the three cases shown spanning seven orders of magnitude in the ratio of the product formation rate constant $\left(k_{\mathrm{p}}=0.1\right)$ to those of the other reaction pathways emanating from zwitterion $\mathbf{Z}$. Reaction time is in arbitrary units depending on the units chosen for the rate constants. (a) Percent conversion to product $\mathbf{P}$; (b) percent of catalyst present as zwitterion $\mathbf{Z}$ as a function of conversion to product $\mathbf{P}$.

Strikingly, Fig. 1 shows that for a given value of $k_{\mathrm{p}}$, identical temporal kinetic profiles are obtained for all cases, regardless of whether the product formation rate constant $k_{\mathrm{p}}$ is much smaller or much larger in value than the rate constants for the other reactions of the zwitterion $\mathbf{Z}$ (Fig. 1a). Zwitterion concentration decreases linearly with increasing conversion to product and is independent of the relative values of the rate constants of the other reactions of the zwitterion, $k_{\mathbf{S M}}$ or $k_{\mathbf{S I}}$ (Fig. 1b). These results show that the rate of product formation $\mathbf{P}$ is independent of the relative rates of zwitterion dissociation back to starting materials or cyclization to form the stable off-cycle intermediate.

A second set of simulations was carried out employing the values of $k_{\mathbf{S I}}$ and $k_{\mathbf{S M}}$ from case 3 in Scheme 6 and Fig. 1, but with the value of $k_{\mathrm{p}}$ now varied over two orders of magnitude. The results of these simulations are shown in Fig. 2, demonstrating that, as expected, the product formation rate is 

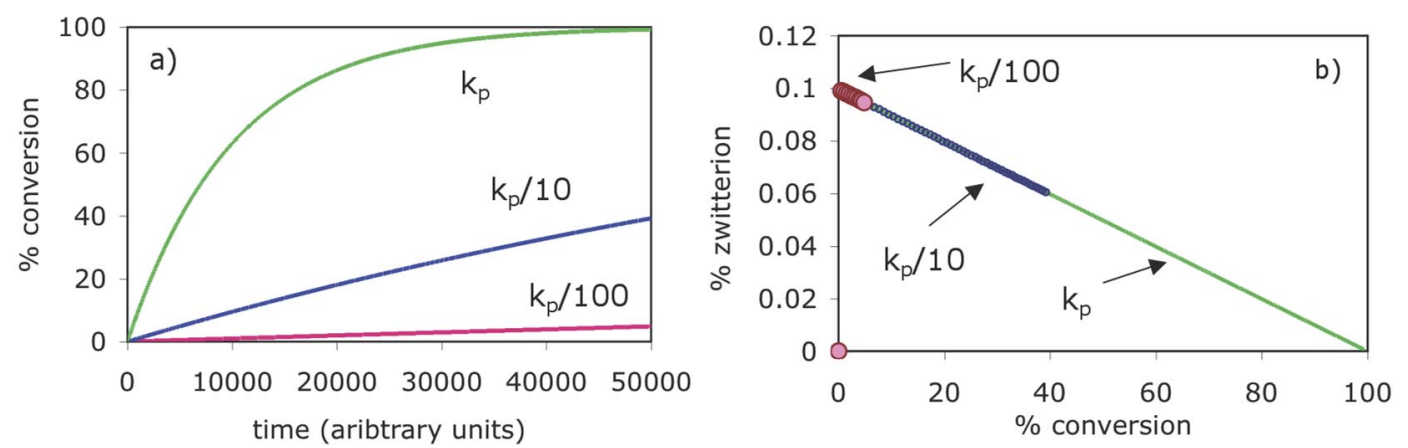

Fig. 2 Kinetic simulations of the reaction network shown in Scheme 6 for case 3 with the product formation rate constant $k_{\mathrm{p}}$ together with simulations in which $k_{\mathrm{p}}$ is decreased over two orders of magnitude. Reaction time is in arbitrary units depending on the units chosen for the rate constants. (a) Percent conversion to product P; (b) percent of catalyst present as zwitterion $\mathbf{Z}$ as a function of conversion to product $\mathbf{P}$, with profiles plotted from reaction initiation up to the conversion attained at the final time shown in Fig. 2a.

slowed when $k_{\mathrm{p}}$ is decreased while keeping all other parameters constant. However, Fig. 2b shows that the zwitterion concentration $[\mathbf{Z}]$ at any given conversion level remains identical for all values of $k_{\mathrm{p}}$. These results again demonstrate that the rate of the reaction to form product $\mathbf{P}$ is independent of the rate constants for the other reversible reactions of the zwitterion. This is quantified by considering the elementary rate law for product formation given by eq. 1 . Product formation depends only on the rate constant $k_{\mathrm{p}}$ and the concentration driving force $[\mathbf{Z}](t)$ and is not influenced by the relationship between $k_{\mathrm{p}}$ and other zwitterion reaction rate constants.

$$
\operatorname{rate}(\mathbf{P})=k_{\mathrm{p}} \cdot[\mathbf{Z}](t)
$$

All reactions of the common zwitterion depend equally on its concentration. Thus, an observed lack of reactivity specifically towards product—but not towards other pathways in these reaction networks - cannot be attributed to low zwitterion concentration. This highlights a critical feature of such networks: if a zwitterion intermediate $\mathbf{Z}$ is a common reactant in several distinct reaction pathways and is in reversible equilibrium with the other species in the network, its temporal concentration $[\mathbf{Z}](t)$ will be dictated only by the thermodynamics of these equilibria. Consideration of the selectivity of pathways leading from the common zwitterion must focus on the relative rate constants and not on the zwitterion concentration.

These simulations lead to the general conclusion that the failure to observe nitroaldehyde product in a reaction scheme such as that of Scheme 4, which is based on the experimental work of Seebach and co-workers for the general network in Scheme 6, may be attributed to the "missing reactivity" of the zwitterion $\mathbf{Z}$ specifically toward product formation, even while this species remains reactive towards-and in dynamic equilibrium with-other species in the network. As long as formation of an off-cycle intermediate from $\mathbf{Z}$ is not irreversible under the conditions of the experiment, then lack of catalyst turnover cannot be attributed to the low concentration of $\mathbf{Z}$. This holds important implications for catalyst and reaction design: observation of reversibility in the formation of downstream species makes it possible to explore specific means to promote reactivity of the zwitterion towards the product, as in fact, has been observed experimentally by addition of additives such as acids. If, by contrast, an off-cycle species is irreversibly formed, then it may be suggested that optimization of the reaction for efficient catalysis should focus on finding a means of avoiding this reaction rather than directly promoting the product-forming step. 


\section{Competitive catalytic reaction networks involving zwitterions}

In analogy to the stoichiometric networks of Scheme 4 studied by Seebach and co-workers, the competitive catalytic network of Scheme 5 is proposed to include zwitterionic species as the first intermediate formed upon reaction of enamines and nitro-olefins. Scheme 7 outlines a general competitive network involving two aldehydes $\mathbf{A}$ and $\mathbf{B}$ whose respective enamines react with the same nitro-olefin to form zwitterions $\mathbf{Z}_{\mathbf{A}}$ and $\mathbf{Z}_{\mathbf{B}}$ in reversible reactions. The zwitterions may cyclize to form stable cyclobutane intermediates $\mathbf{S I}_{\mathbf{A}}$ and $\mathbf{S I}_{\mathbf{B}}$ in reversible reactions, and they may react to form nitroaldehyde products.

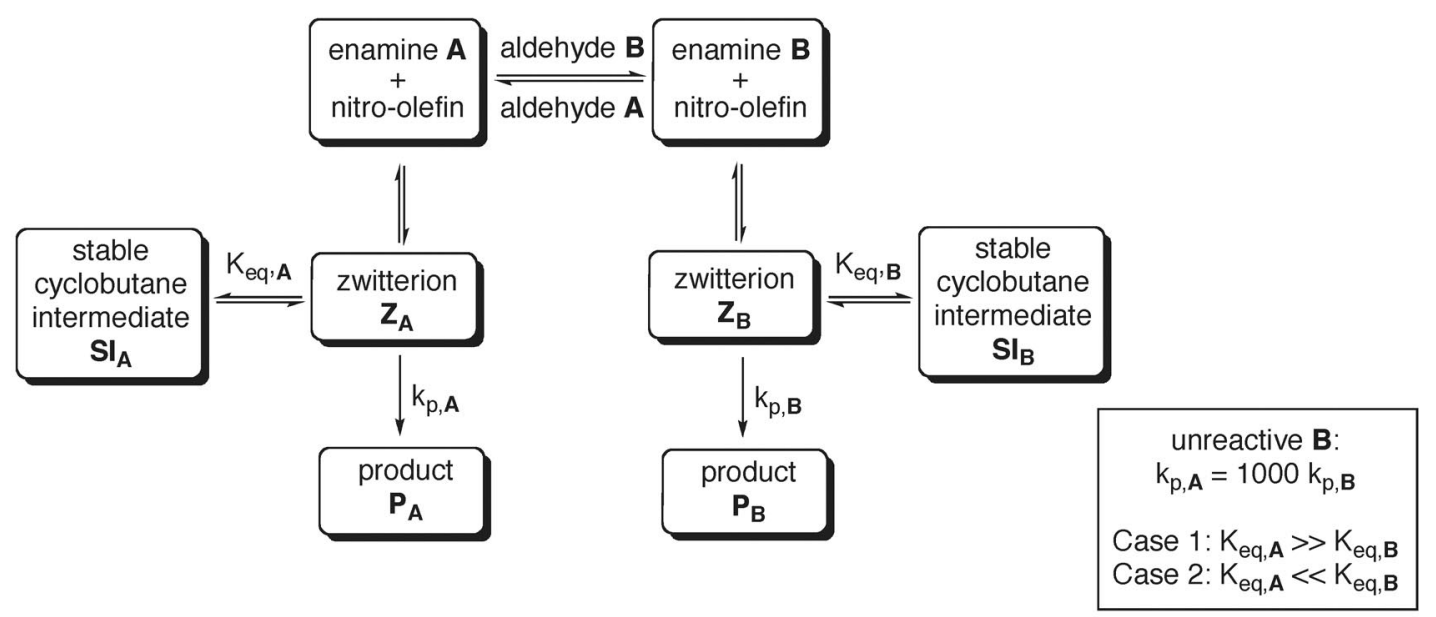

Scheme 7 Competitive reaction network with the enamines of two aldehydes $\mathbf{A}$ and $\mathbf{B}$ reacting reversibly with a nitro-olefin to form their respective zwitterions $\mathbf{Z}_{\mathbf{A}}$ and $\mathbf{Z}_{\mathbf{B}}$, their respective stable cyclobutane intermediates $\mathbf{S I}_{\mathbf{A}}$ and $\mathbf{S I}_{\mathbf{B}}$, and their respective reaction products $\mathbf{P}_{\mathbf{A}}$ and $\mathbf{P}_{\mathbf{B}}$ (with regeneration of the catalyst, not shown). Simulations are carried out for the case where one product pathway is unreactive, varying the relative stability of the two off-cycle intermediates.

Kinetic simulations of this reactant network were carried out under a number of different conditions outlined in Scheme 7. We treat the case where aldehydes $\mathbf{A}$ and $\mathbf{B}$ form enamines and zwitterions reversibly and with equal stability, but where one of the aldehydes ( $\mathbf{B}$ in this case) is much less reactive towards product formation $\left(k_{\mathrm{p}, \mathbf{A}}>>k_{\mathrm{p}, \mathbf{B}}\right)$. The zwitterions also form stable, off-cycle cyclobutane intermediates, and we treat two cases: one in which the cyclobutane $\mathbf{S I}_{\mathbf{A}}$ formed from $\mathbf{A}$ is much more stable than that formed from $\mathbf{B}$, and the opposite case where $\mathbf{S I}_{\mathbf{A}}$ is much less stable than $\mathbf{S I}_{\mathbf{B}}$. These scenarios probe the role of stable off-cycle intermediates in competitive reactions where one pathway is significantly less reactive to form product than the other.

The results of these simulations are shown in Fig. 3. Conversion of aldehyde $\mathbf{A}$ to form product $\mathbf{P}_{\mathbf{A}}$ is complete before any product $\mathbf{P}_{\mathbf{B}}$ is formed from the more inert aldehyde $\mathbf{B}$. The reaction exhibits zero-order kinetics in substrate concentrations for the case where the catalyst is sequestered as the stable, off-cycle cyclobutane on the active $\left(\mathbf{P}_{\mathbf{A}}\right)$ side of the network, resembling the kinetic profiles observed experimentally for this reaction with linear aldehydes. Interestingly, simulations show that when the resting state is the cyclobutane species formed from aldehyde $\mathbf{B}$, which is inactive in forming product, product $\mathbf{P}_{\mathbf{A}}$ formation from the linear aldehyde $\mathbf{A}$ is considerably slower and exhibits firstorder kinetics. In the presence of the unreactive aldehyde $\mathbf{B}$, formation of $\mathbf{S I}_{\mathbf{B}}$ as the resting state serves to decrease the active catalyst concentration available to the active side of the network, which proceeds in the absence of $\mathbf{S I}_{\mathbf{A}}$, albeit more slowly. The final conversion of $\mathbf{A}$ occurs with nitrostyrene released via dissociation of $\mathbf{S I}_{\mathbf{B}}$. This result confirms that the lack of reactivity observed experimentally for 

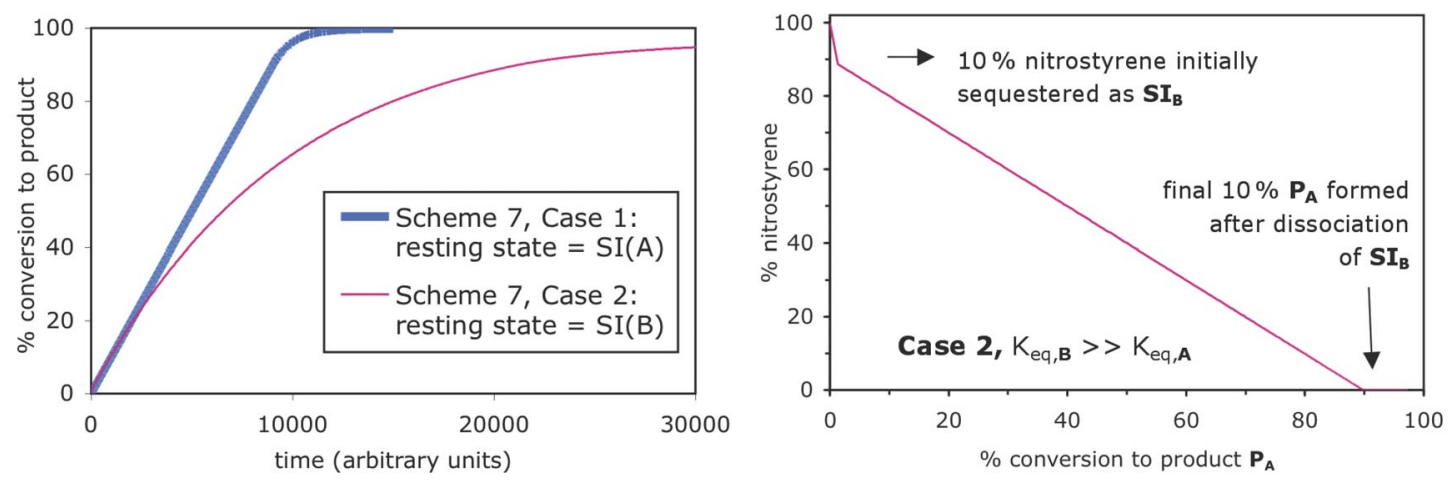

Fig. 3 Simulation of reaction network of Scheme 7 carried out with equimolar amounts of aldehydes $\mathbf{A}$ and $\mathbf{B}$ and nitrostyrene, with $10 \mathrm{~mol} \%$ catalyst. Left: Conversion to product $\mathbf{P}_{\mathbf{A}}$ as a function of time for cases 1 and 2, where the catalyst is sequestered as stable intermediate $\mathbf{S I}_{\mathbf{A}}$ and $\mathbf{S I}_{\mathbf{B}}$, respectively. Right: Consumption of nitrostyrene as a function of product formation for case 2, where the catalyst is sequestered as stable intermediate $\mathbf{S I}_{\mathbf{B}}$. The reactivity of $\mathbf{S I}_{\mathbf{B}}$ for ring-opening is demonstrated by the last $10 \%$ conversion to product, which occurs after dissociation of $\mathbf{S I}_{\mathbf{B}}$ frees up nitrostyrene for reaction on the $\mathbf{A}$ side of the network.

$\alpha$-substituted aldehydes cannot be explained by the failure of the stable cyclobutane intermediates to ring-open.

The origin of the very different reactivity towards nitroaldehyde product from linear and $\alpha$-substituted aldehydes bears further discussion. The disparate reactivities are difficult to rationalize solely by considering the zwitterion depicted in Scheme 2 . Since the presence of $R^{2} \neq H$ hinders neither the formation of aldehyde enamine nor its reaction with nitro-olefins to form cyclobutanes, both of which proceed in rapid, reversible fashion, significant differences in the further reactions of the respective zwitterions $\left(\mathrm{R}^{2}=\mathrm{H}\right.$ vs. $\left.\mathrm{R}^{2} \neq \mathrm{H}\right)$ toward the nitroaldehyde product (protonation, deprotonation, or hydrolysis) are difficult to explain. We proposed that this difference in reactivity may be explained if linear and branched aldehydes proceed via different pathways, as shown in Scheme 8. Based on our finding of kinetic stereospecificity in the formation of stable nitroaldehyde product enamines $\mathbf{8}$ from linear aldehydes - species that cannot be formed from $\alpha$-substituted aldehydes - the difference in linear vs. branched aldehyde activity may be attributed to a pathway that passes through the cyclobutane $\mathbf{7}$ and product enamine $\mathbf{8}$ for linear aldehydes. Cyclobutanes formed from linear aldehydes may undergo deprotonation/protonation, possibly via the 1,2- $\mathrm{N}$-oxazine $\mathbf{6}$ (observed experimentally as a minor species in equilibrium with the cyclobutane for linear aldehydes only) to form the enamine 8 . At this point this novel route rejoins the conventional route with $\mathbf{8}$ undergoing hydrolysis to $\mathbf{9}$ and the product nitroaldehyde 3. Interestingly, in contrast to the mechanism of Scheme 6, where the zwitterion concentration could not be used to rationalize different reactivities of pathways emanating from this common species, in the case depicted in Scheme 8 it is the low concentration of $\mathbf{Z}$ compared to $\mathbf{7}$ that is key to explaining the difference in reactivity of two parallel pathways emanating from the two species. The steps through cyclobutane $\mathbf{7}$ and product enamine $\mathbf{8}$ may be much faster than the direct hydrolysis of the zwitterion $\mathbf{Z}$, due simply to the much greater concentration driving force of [7] compared to [Z], since nearly $100 \%$ of the catalyst sits as $\mathbf{7}$ as the resting state. This highlights a critical implication of species such as 7 being on rather than off the catalytic cycle. 


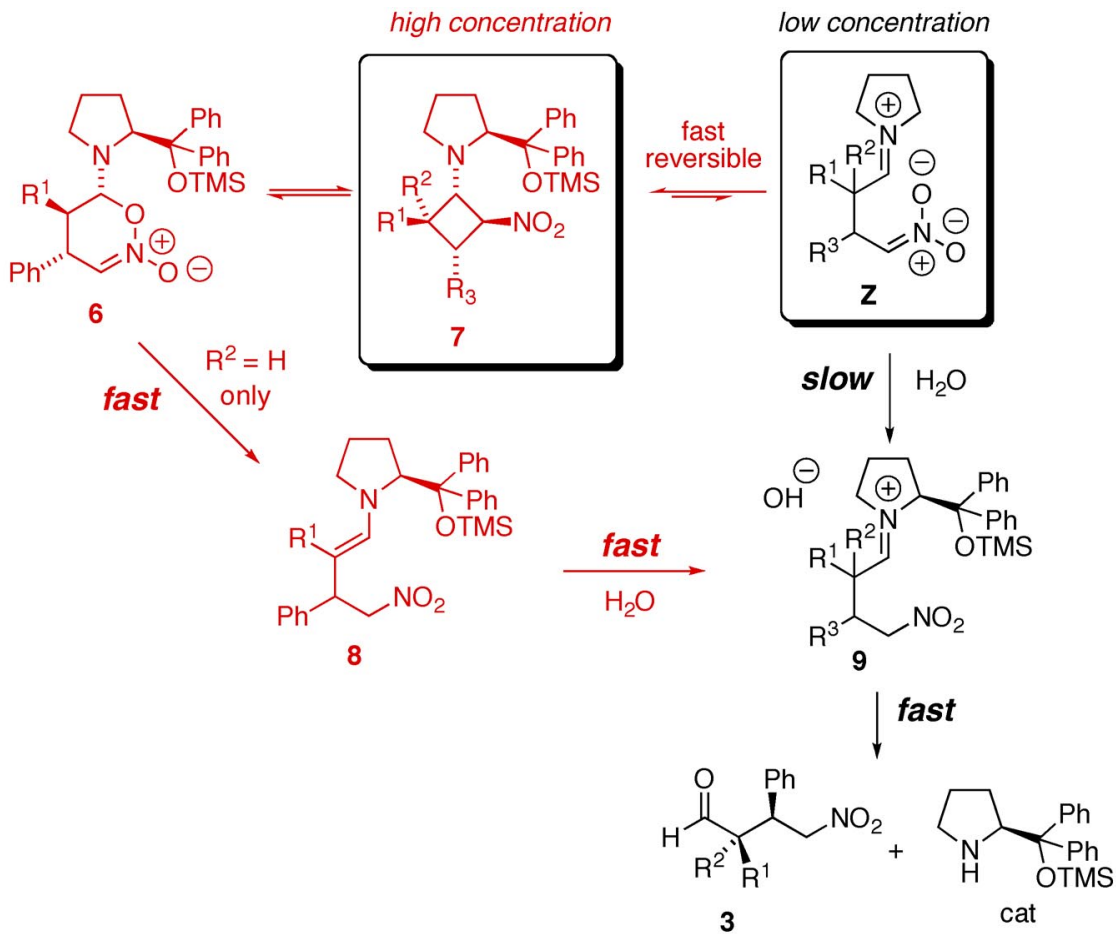

Scheme 8 Alternate reaction pathway depicted on the left side of the scheme via cyclobutane 7 (in high concentration) that is proposed to operate in parallel with the pathway directly through zwitterion $\mathbf{Z}$ (in low concentration) for linear aldehydes. The sluggish activity observed for $\alpha$-substituted compared to linear aldehydes is rationalized because the novel pathway via a species such as $\mathbf{8}$ is not available for the latter but is highly competitive for the former.

\section{Enantioselective catalytic reaction networks involving zwitterions}

The results shown above shed some light on the relative role of reactivity and stability of stable intermediates in competitive reactions between two different substrates. A more pertinent type of competition is that faced by intermediates in asymmetric catalytic transformations where a single set of substrates may form different enantiomers or diastereomers. The role of stable downstream intermediates may be explored in such cases, as outlined in Schemes 9 and 10. In these scenarios, we see again that the position of the stable downstream species-whether it lies on or off the catalytic cycle-is key to rationalizing both reactivity and selectivity.

Schemes 9 and 10 show two different pathways to product. The conventional catalytic pathway with the cyclobutane species occurring off-cycle is shown with gray arrows. The novel pathway proposing that these downstream intermediates lie on the cycle is shown at the outside of the scheme. It is worth noting that both the conventional pathway and the novel pathway pass through zwitterion intermediate species $\mathbf{Z}_{\mathbf{A}}$ and $\mathbf{Z}_{\mathbf{B}}$, with facial selectivity (as described in Scheme 2) being determined by the ratio of the equilibrium constants $K_{\text {fac }}$ and $K_{\text {ent-fac }}$ for the reversible attack of the enamine on the electrophile to form the zwitterions. $\mathbf{Z}_{\mathbf{A}}$ and $\mathbf{Z}_{\mathbf{B}}$ go on to form stable cyclobutane intermediates $\mathbf{S I}_{\mathbf{A}}$ and $\mathbf{S I}_{\mathbf{B}}$ reversibly. This scenario represents the full catalytic cycle encompassing both facial selectivity at the stereogenic bond-forming step and the possibility of parallel reaction pathways directly through the zwitterion (off-cycle pathway) or through the stable cyclobutane intermediate (on-cycle pathway). For simplicity, we assume that only one cyclobutane diastereomer is formed on either side of the network 


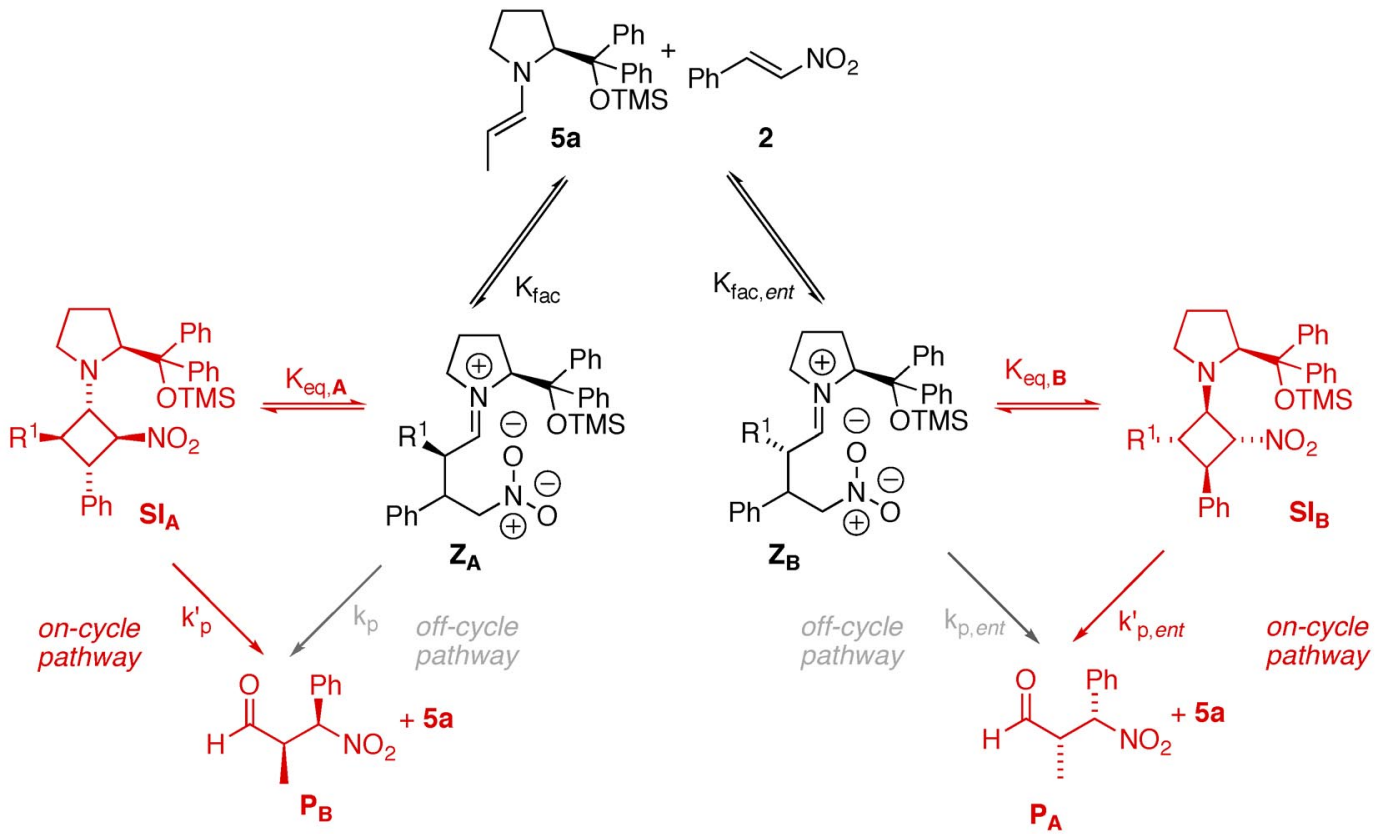

Scheme 9 Catalytic cycles with two alternate pathways as in Scheme 8 but including facial selectivity to two enantiomeric zwitterions. Pathways at the outside of the scheme show the enantiomeric cyclobutane species $\mathbf{S I}_{\mathbf{A}}$ and $\mathbf{S I}_{\mathbf{B}}$ being directly on the catalytic cycle to products $\mathbf{P}_{\mathbf{A}}$ and $\mathbf{P}_{\mathbf{B}}$, respectively. Gray arrows show the conventional pathway to products from the zwitterions with the cyclobutane species $\mathbf{Z}_{\mathbf{A}}$ and $\mathbf{Z}_{\mathbf{B}}$ as off-cycle intermediates.



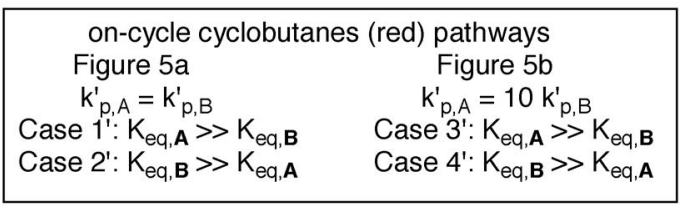

Scheme 10 Networks for simulations of enantiomeric catalytic cycles shown in Scheme 9. Gray arrows show reaction to product along the conventional pathways from the zwitterions. Pathways at the outside of the scheme show the new proposed pathways through the cyclobutane species. For both networks, simulations are carried out for two ratios of the product formation rate constant and for cases where each cyclobutane intermediate serves as the resting state. 
(opposite configuration at all centers except that of the catalyst). In addition, we assume perfect diastereoselectivity to the syn product in all cases.

Further steps to product along the conventional route are collapsed as the rate-determining $k_{\mathrm{p}, \mathbf{A}}$ and $k_{\mathrm{p}, \mathbf{B}}$ shown with gray arrows. In this scenario, the cyclobutane species lie off the catalytic cycle, connected as reversible reservoirs of stable intermediates. For the "on-cycle" route shown at the outside of the scheme, the pathway to product passes through the cyclobutane species rather than considering it as an off-cycle intermediate. Again, further steps to product are collapsed in the simulations as the rate-determining $k_{\mathrm{p}, \mathbf{A}}^{\prime}$ and $k_{\mathrm{p}, \mathbf{B}}^{\prime}$. Our simulations explore cases where the stable resting state sequestering most of the catalyst concentration occurs on each side of the network.

For both the on- and off-cycle scenarios described in Schemes 9 and 10, our simulations explore how enantioselectivity is influenced by the following factors: (i) facial selectivity in formation of the zwitterions, $K_{\text {fac }}$ vs. $K_{\text {ent-fac}}$; (ii) the relative stability of the two cyclobutane intermediates either on or off the cycle, $K_{\text {eq, } \mathbf{A}}$ vs. $K_{\text {eq, }, \mathbf{B}}$; and (iii) the relative rate constants for product formation, either from the cyclobutanes $\left(k_{\mathrm{p}, \mathbf{A}}^{\prime}\right.$ vs. $\left.k_{\mathrm{p}, \mathbf{B}}^{\prime}\right)$ or from the zwitterions $\left(k_{\mathrm{p}, \mathbf{A}}\right.$ vs. $\left.k_{\mathrm{p}, \mathbf{B}}\right)$.

\section{Conventional (off-cycle) pathway}

Figure 4 presents results of simulations for the case shown with gray arrows in Schemes 9 and 10, where the cyclobutane species occurs off of the catalytic cycle. Figure 4a shows product enantioselectivity for four different levels of facial selectivity in the addition of the enamine to nitrostyrene for the case where the product formation rate is identical for each side of the network $\left(k_{\mathrm{p}, \mathbf{A}}=k_{\mathrm{p}, \mathbf{B}}\right)$. The simulations treat the case where the resting state is the cyclobutane $\mathbf{S I}_{\mathbf{A}}\left(K_{\mathrm{eq}, \mathbf{A}}>>\mathrm{K}_{\mathrm{eq}, \mathbf{B}}\right.$, solid bars $)$ and the case where the resting state is $\mathbf{S I}_{\mathbf{B}}\left(\left(K_{\mathrm{eq}, \mathbf{B}}>>K_{\mathrm{eq}, \mathbf{A}}\right.\right.$, hatched bars $)$. Figure $4 \mathrm{~b}$ presents results of similar simulations where the ratio of product formation rate constants is $k_{\mathrm{p}, \mathbf{A}}=k_{\mathrm{p}, \mathbf{B}}=10: 1$. Figure $4 \mathrm{a}$ shows that when the network exhibits neither facial selectivity at the nitrostyrene addition step nor selectivity at the product formation step, the reaction gives racemic product regardless of the relative stability of off-cycle cyclobutanes. Increasing facial selectivity gives increased product enantioselectivity, but again the result is independent of whether the catalyst resting state is on the side of the network with high facial selectivity or on the side with low selectivity. Figure $4 \mathrm{~b}$ shows that increasing selectivity in the product formation step gives increasing enantioselectivity. High facial selectivity and high product formation selectivity combine to give the highest enantiomeric excesses. Strikingly, the position of the resting state of the catalyst, sequestering the majority of the catalyst as either $\mathbf{S I}_{\mathbf{A}}$ or $\mathbf{S I}_{\mathbf{B}}$, has no influence on
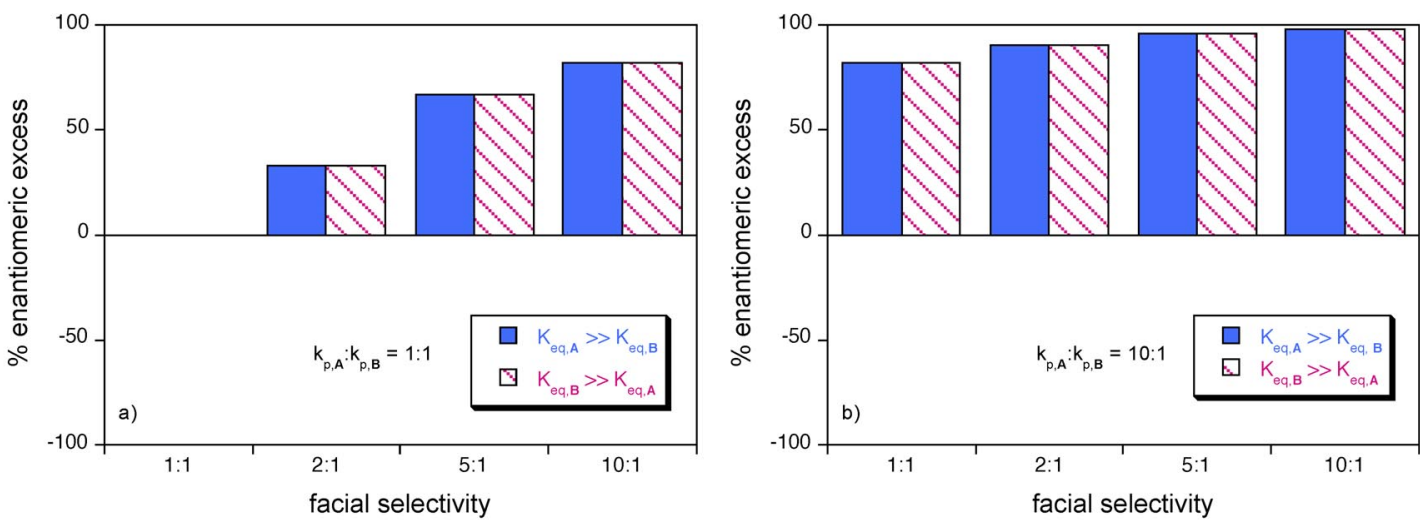

Fig. 4 Product enantiomeric excess $(\%)$ as a function of facial selectivity (A:B) for the reactions outlined in Schemes 9 and 10 for the conventional pathway where the stable cyclobutanes $\mathbf{S I}_{\mathbf{A}}$ and $\mathbf{S I}_{\mathbf{B}}$ occurring off-cycle. Left: product rate constant ratio $k_{\mathrm{p}, \mathbf{A}}: k_{\mathrm{p}, \mathbf{B}}=1: 1$; case 1, solid bars: $\mathbf{S I}_{\mathbf{A}}$ as resting state; case 2, hatched bars: $\mathbf{S I}_{\mathbf{B}}$ as resting state. Right: product rate constant ratio $k_{\mathrm{p}, \mathbf{A}}: k_{\mathrm{p}, \mathbf{B}}=10: 1$; case 3 , solid bars: $\mathbf{S I}_{\mathbf{A}}$ as resting state; case 4, hatched bars: $\mathbf{S I}_{\mathbf{B}}$ as resting state. 
the product enantiomeric excess in any case. These results demonstrate that the downstream cyclobutane intermediate has no effect on product selectivity if it lies off of the catalytic cycle.

\section{Novel (on-cycle) pathway}

Figure 5 presents results of simulations for the case shown by the pathways at the outside of Schemes 9 and 10, where the cyclobutane species appear as species on the active catalytic cycle. In contrast to the results for the conventional pathway presented in Fig. 4, it is clear that the relative stability of the catalyst resting state has a significant impact on the product selectivity when it lies on the cycle. When the majority of the catalyst concentration is sequestered as one of the cyclobutane species, its concentration dictates the product enantiomeric excess, regardless of either facial selectivity in the electrophile addition step or selectivity in the product formation step, and dictated only by which cyclobutane species dominates. Indeed, high facial selectivity can be completely overruled by high stability of the cyclobutane species on the other side of the network. A network with 10:1 facial selectivity towards the $\mathbf{P}$ side of the network gives high enantiomeric excess towards the opposite product when the cyclobutane on the opposite side is more stable than that on the side dictated by the facial selectivity step. Only at very high facial selectivity and very high product formation selectivity does the strong bias towards the opposite cyclobutane intermediate begin to be countered. Similarly, product selectivity in a system exhibiting neither facial selectivity in the electrophile addition step nor selectivity in the product formation step will be completely dictated by the relative stability of the cyclobutane intermediates on the two sides of the network.
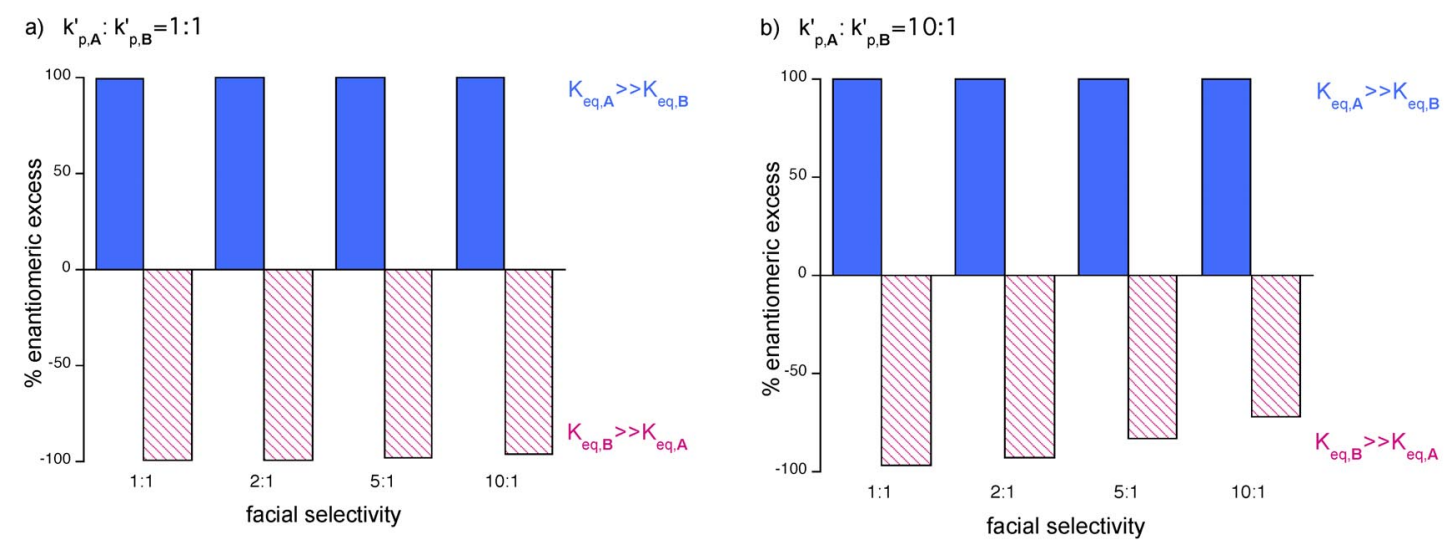

Fig. 5 Product enantiomeric excess $(\%)$ as a function of facial selectivity (A:B) for the reactions outlined in Schemes 9 and 10 for the novel pathway where the stable cyclobutanes $\mathbf{S I}_{\mathbf{A}}$ and $\mathbf{S I}_{\mathbf{B}}$ occurring on-cycle. Left: product rate constant ratio $k_{\mathrm{p}, \mathbf{A}}^{\prime}: k_{\mathrm{p}, \mathbf{B}}^{\prime}=1: 1$; case 1', solid bars: $\mathbf{S I}_{\mathbf{A}}$ as resting state; case 2', hatched bars: $\mathbf{S I}_{\mathbf{B}}$ as resting state. Right: product rate constant ratio $k_{\mathrm{p}, \mathbf{A}}^{\prime}: k_{\mathrm{p}, \mathbf{B}}^{\prime}=10: 1$; case 3 ', solid bars: $\mathbf{S I}_{\mathbf{A}}$ as resting state; case $4^{\prime}$ hatched bars: $\mathbf{S I}_{\mathbf{B}}$ as resting state.

The results of these simulations of the networks shown in Schemes 9 and 10 highlight a number of important implications for reactions where stable downstream species are observed. Clearly, the question of whether such species lie on or off the catalytic cycle is important for understanding their role in both reactivity and selectivity. When downstream species lie off of the catalytic cycle, their presence may influence the overall observed reactivity by decreasing the active catalyst concentration, but as Fig. 4 shows, off-cycle species have no influence on product enantioselectivity, regardless of whether most of the catalyst concentration rests off-cycle on the major or minor product side of the network. This conclusion is dramatically altered if the downstream species lie on the cycle. In such cases, the rel- 
ative stability of downstream species plays a significant role in ultimate product selectivity, regardless of the facial selectivity inherent in the attack of the enamine on the electrophile.

These findings may help to inform catalyst design. Rather than simply considering the transitionstate model of Scheme 2, the relative stability of potential downstream intermediates may be employed as a catalyst design tool. It may be considered likely that the steric considerations that inform the transition state of Scheme 2 - and thus facial selectivity - may similarly apply to considerations of the relative stability of downstream intermediates; if so, the dramatic results of the solid bars in Fig. 5 may serve to outline the limiting cases rather than representing practical examples. Most importantly, these simulations demonstrate that each level in the catalytic network has the potential to dictate selectivity. Nuances in the choice of catalyst substituents that impact the resulting intermediate structures differently might play an important role in ultimate product selectivity.

The imperative for distinguishing between on- and off-cycle downstream intermediates becomes particularly evident in the case of the "benchmark" reaction of Scheme 1, the conjugate addition of nitro-olefins. While the diphenylprolinol ether catalyst 3 routinely achieves $>98 \%$ ee in this reaction, employing different electrophiles with the same enamine 5a often yields lower selectivities. For example, amination of 5a with DEAD yields $79 \%$ ee [2c]. Interestingly, the kinetic profile of that reaction suggests that no downstream intermediates are formed in that case and that the reaction between enamine and electrophile is irreversible, meaning that the enantio-determining step may be considered to be as depicted in the conventional transition-state model of Scheme 2. Given this analysis, perhaps $79 \%$ ee should be considered as the "benchmark" facial selectivity for an X=Y electrophile following the steric transition state model of Scheme 2 . The lower selectivity of the reaction with DEAD in fact may represent the true facial selectivity of enamine 5a towards general electrophiles $\mathrm{X}=\mathrm{Y}$ in irreversible reactions. By contrast, the simulation results of Fig. 5 support the suggestion that the near-perfect enantioselectivity observed for nitro-olefins as electrophiles may not represent a benchmark of the step shown in Scheme 2, but could in fact be due to the role of the stable cyclobutane species reversibly equilibrated with upstream species and lying directly on the catalytic cycle. Thus, the intrinsic selectivity of this transition state would be enhanced by the contribution of the on-cycle, downstream, reversibly formed cyclobutane in a way that may benefit a variety of catalysts in this particular reaction but that is not general for reactions of other electrophiles. The designation of the reaction of Scheme 1 as a benchmark for testing the intrinsic selectivity of different catalysts according to the transition-state model of Scheme 2 may thus be called into question.

\section{CONCLUSIONS}

Kinetic modeling of stoichiometric and catalytic reaction networks has shed light on the mechanism and role of intermediates in organocatalytic reactions using diarylprolinol ether catalysts. These studies help to address the role of intermediates formed downstream from the step generally considered to be enantio-differentiating in enamine catalysis. The central role of zwitterionic intermediates formed from the interaction between enamines and nitro-olefins is addressed. When such a species is common to several different reaction pathways, its low concentration cannot be invoked to explain differences in relative reactivities of these pathways. Thus, when downstream intermediates are connected reversibly but off-cycle to such zwitterions, the relative stability of these off-cycle intermediates cannot influence selectivity on the catalytic cycle. By contrast, if downstream intermediates lie on the catalytic cycle, they may represent a parallel reaction pathway in competition with pathways through the zwitterion. In such cases, the relative stability of these intermediates can have a significant effect on the stereochemical outcome of the reaction. These results may help to stimulate ideas for catalyst design, and they highlight the critical importance of distinguishing on- and off-cycle species for increasing mechanistic understanding and practical reaction optimization. 


\section{SUPPLEMENTARY INFORMATION}

Supplementary information is available online (http://dx.doi.org/10.1351/PAC-CON-13-01-14).

\section{REFERENCES AND NOTES}

1. (a) M. B. Schmid, K. Zeitler, R. M. Gschwind. Angew. Chem., Int. Ed. 49, 4997 (2010); (b) M. B. Schmid, K. Zeitler, R. M. Gschwind. J. Am. Chem. Soc. 133, 7065 (2011); (c) M. B. Schmid, K. Zeitler, R. M. Gschwind. Chem. Sci. 2, 1793 (2011).

2. (a) J. Burés, A. Armstrong, D. G. Blackmond. J Am. Chem. Soc. 133, 8822 (2011); (b) J. Burés, A. Armstrong, D. G. Blackmond. J. Am. Chem. Soc. 133, 6741 (2012); (c) J. Burés, A. Armstrong, D. G. Blackmond. Chem. Sci. 3, 1273 (2012).

3. (a) K. Patora-Komisarska, M. Benohoud, H. Ishikawa, D. Seebach, Y. Hayashi. Helv. Chim. Acta 94, 719 (2011); (b) D. Seebach, X. Sun, C. Sparr, M.-O. Ebert, W. B. Schweizer, A. K. Beck. Helv. Chim. Acta 95, 1064 (2012); (c) D. Seebach, X. Sun, M.-O. Ebert, W. B. Schweizer, N. Purkayastha, A. K. Beck, J. Duschmale, H. Wennemers, T. Mukaiyama, M. Benohoud, Y. Hayashi, M. Reiher. Helv. Chim. Acta 96, 799 (2013).

4. (a) S. J. Blarer, D. Seebach. Chem. Ber. 116, 3086 (1983); (b) D. Seebach, A. K. Beck, J. Golinski, J. N. Hay, T. Laube. Helv. Chim. Acta 68, 162 (1985); (c) D. Seebach, J. Golinski. Helv. Chim. Acta 64, 1413 (1981).

5. (a) K. C. Brannock, A. Bell, R. D. Burpitt, C. A. Kelly. J. Org. Chem. 29, 801 (1964); (b) M. E. Kuehne, L. Foley. J. Org. Chem. 30, 4280 (1965); (c) A. Risaliti, M. Forchiassin, E. Valentin. Tetrahedron Lett. 7, 6331 (1966); (d) H. Feuer, A. Hirschfeld, E. D. Bergmann. Tetrahedron. 24, 1187 (1968); (e) A. T. Nielsen, T. G. Archibald. Tetrahedron 26, 3475 (1970); (f) E. Valentin, G. Pitacco, F. P. Colonna. Tetrahedron Lett. 13, 2837 (1972); (g) F. P. Colonna, E. Valentin, G. Pitacco, A. Risaliti. Tetrahedron 29, 3011 (1973); (h) F. Felluga, P. Nitti, G. Pitacco, E. Valentin. Tetrahedron 45, 5667 (1989); (i) F. Felluga, P. Nitti, G. Pitacco, E. Valentin. J. Chem. Soc., Perkin Trans. 12331 (1992).

6. Y. Hayashi, H. Gotoh, T. Hayashi, M. Shoji. Angew. Chem., Int. Ed. 44, 4212 (2005).

7. J. Franzen, M. Marigo, D. Fielenbach, T. C. Wabnitz, A. Kjaersgaard, K.-A. Jorgensen. J. Am. Chem. Soc. 127, 18296 (2005).

8. C. Moberg. Angew. Chem., Int. Ed. 52, 2160 (2013).

9. K. L. Jensen, G. Dickmeiss, H. Jiang, L. Albrecht, K.-A. Jorgensen. Acc. Chem. Res. 45, 248 (2012).

10. The term "resting state" refers to the catalytic species under reaction conditions that contains the dominant fraction of the catalyst initially added to the reaction mixture. This species may be identified by kinetic experiments and it may reside on or off the active catalytic cycle.

11. G. Sahoo, H. Rahaman, A. Madarasz, I. Papai, M. Melarto, A. Valkonen, P. M. Pihko. Angew. Chem., Int. Ed. 51, 8495 (2012).

12. (a) C. R. Landis, J. A. Halpern. J. Am. Chem. Soc. 109, 1746 (1987); (b) J. Halpern. Science 217, 401 (1982).

13. A. C. Ferretti, J. S. Mathew, I. Ashworth, M. Purdy, C. Brennan, D. G. Blackmond. Adv. Synth. Catal. 350, 1007 (2008).

14. IUPAC. Compendium of Chemical Terminology, $2^{\text {nd }}$ ed. (the "Gold Book"). Compiled by A. D. McNaught and A. Wilkinson. Blackwell Scientific Publications, Oxford (1997). XML on-line corrected version: http://dx.doi.org/10.1351/goldbook (2006-) created by M. Nic, J. Jirat, B. Kosata; updates compiled by A. Jenkins; "Curtin-Hammett principle": doi:10.1351/goldbook.C01480. 
15. Kinetic simulations carried out using Copasi v. 4.6, see www.copasi.org; S. Hoops, S. Sahle, R. Gauges, C. Lee, J. Pahle, N. Simus, M. Singhal, L. Xu, P. Mendes, U. Kummer. Bioinformatics 22, 3067 (2006). 